\title{
Energetics and electronic structures of polymeric all-benzene hollow-cage and planar networks
}

\author{
Masaki Mieda and Susumu Okada* \\ Graduate School of Pure and Applied Sciences, University of Tsukuba, 1-1-1 Tennodai, Tsukuba, Ibaraki \\ 305-8571, Japan
}

\begin{abstract}
We studied the energetics and electronic structure of polymerized benzene hollow-cages and sheet using the density functional theory with the generalized gradient approximation. The energetics and electronic structure of the polymeric benzene cages and sheet depend on their size and dimensionality. Because of the symmetric network topology as well as the constituent benzene units, the cages possess highly bunched states around the Fermi level. The energy gap between the highest occupied and the lowest unoccupied states of the cages are approximately proportional to their curvature, owing to the decrease of the strain. The polymerized benzene sheet is an direct gap semiconductor with the gap of $2.4 \mathrm{eV}$ between the less dispersive states of the highest brach of the valence and the lowest branch of the conduction bands.
\end{abstract}

\section{Introduction}

Electronic structures of carbon allotropes and hydrocarbon molecules consisting of $\mathrm{sp}^{2}$ $\mathrm{C}$ atoms are sensitive to their network topology and boundary condition, allowing them key materials for wide applications in current and future functional devices. Although graphene is a zero-gap semiconductor with a pair of linear dispersion band at the Fermi level, ${ }^{1,2)}$ hydrocarbon molecules having primarily armchair edges, which are regarded as graphene nanoflakes, possess a basically symmetric electronic structure with respect to the Fermi level and a moderate energy gap between the highest occupied and the lowest unoccupied states, which is inversely proportional to the molecular size. ${ }^{3)}$ Hydrocarbon molecules with triangular shape and zigzag edges possess radical spin of which spin states correspond with an imbalance between the numbers of two sublattices in their networks. ${ }^{4-8)}$ Two-dimensional networks of graphitic $\mathrm{sp}^{2}$ network with topological defects possess magnetic states as their ground state, depending on the defect species and their $\pi$ electron network topology. ${ }^{9-13)}$ In addition, by imposing a boundary condition on graphitic network, resultant materials, i.e. carbon nanotube ${ }^{14-16)}$ and graphene

\footnotetext{
${ }^{*}$ E-mail: sokada@comas.frsc.tsukuba.ac.jp
} 
nanoribbons, ${ }^{17}{ }^{18)}$ exhibit metallic, semiconducting, and magnetic electronic properties, depending on the tiny difference in the topology and boundary condition.

Networks comprising hydrocarbon molecule are subject to study as for the hierarchical nanomaterials of benzene and other hydrocarbon molecules with unusual physical and chemical properties. Hydrocarbon molecules can form various structural morphologies, such as cycloparaphenylene, ${ }^{19-23)}$ cyclometaphenylene, ${ }^{24-29)}$ benzene nanocages, ${ }^{30-33)}$ benzene nanobowl, ${ }^{34,35)}$ and two-dimensional polymer ${ }^{36)}$ exhibiting interesting structural variations. Owing to their structures, they are capable of accommodating foreign atoms and molecules forming complexes with interesting structural hierarchy and unusual electronic structures. ${ }^{37,38)}$ The electronic structure of these nanosucale hydrocarbon materials is sensitive to the constituent species and their conformations in the networks. Indeed, a hexagonal network of phenalenyl and phenyl groups, which are alternately arranged, has both the Dirac cone and the kagome band near the Fermi level. ${ }^{12,13)}$

Thus, in this work, we aim to clarify the energetics and electronic structures of polymerized networks of benzene with zero and two dimensions, as prototypes of the hierarchical nanomaterials of hydrocarbon molecules. Our theoretical calculations based on the density functional theory (DFT) showed that the energetics and electronic structure of the polymeric benzene cages and sheet depend on their size and dimensionality. The energy gap between the highest occupied and the lowest unoccupied states of the cages are proportional to their cage sized, despite the detailed electronic structure is sensitive to the cage. As for the two dimensional network of the polymerized benzene, we found that the flat dispersion bands both at the conduction and valence band edges.

\section{Calculation methods}

All calculations are based on $\mathrm{DFT}^{39,40)}$ as implemented in the program package Simulation Tools for Atom TEchnology (STATE). ${ }^{41)}$ We use the generalized gradient approximation (GGA) with the Perdew-Burke-Ernzerhof functional ${ }^{42)}$ to describe the exchange-correlation potential energy among interacting electrons. Ultrasoft pseudopotentials with the Vanderbilt scheme are adopted as the interaction between electrons and ions. ${ }^{43)}$ Valence wave functions and the deficit charge density are expanded in terms of plane wave basis sets with cutoff energies of 25 and 225 Ry, respectively. Integration over the Brillouin zones of the polymerized benzene cages and sheet were executed by using the $\Gamma$ point and the equidistant $4 \times 4 \times 1 \boldsymbol{k}$-mesh samplings, respectively. Geomet- 


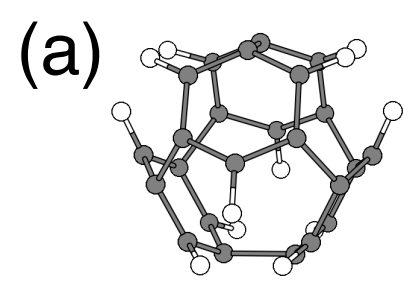

(d)
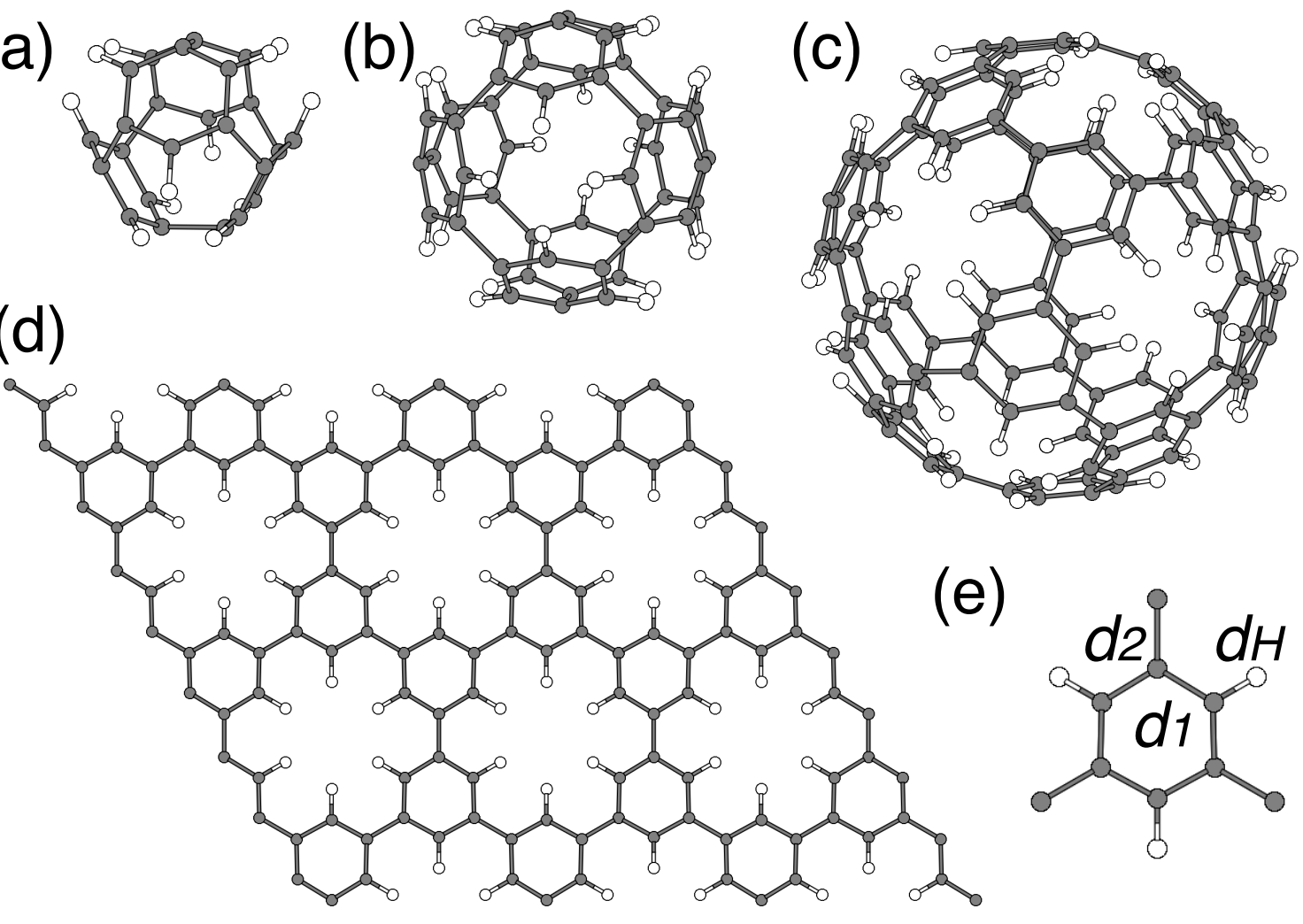

Fig. 1. Optimized geometries of (a) [4]-, (b) [8]-, (c) [20]-benzene cages and (d) that of the benzene polymer. (e) The structural unit of polymerized benzene cages and sheet. Gray and white circles denote $\mathrm{C}$ and $\mathrm{H}$ atoms, respectively.

ric structures of the polymerized benzene cages and sheet are fully optimized until the force acting on each atom is less than $1.33 \times 10^{-3} \mathrm{HR} / \mathrm{au}$. To simulate isolated benzene cages and an isolated two-dimensional hydrocarbon network, each of which is separated by at least $0.6 \mathrm{~nm}$ vacuum spacing to its periodic images. To investigate their thermal stability, first principles molecular dynamics (MD) simulations were conducted using the velocity scaling method to keep the temperature constant.

\section{Results and discussion}

Figure 1 shows optimized structures of the polymerized benzene nanocages and sheet. For the polymerized benzene cages, we consider the cages consisting of 4, 8, and 20 benzene rings with tetrahedral [[4]-benzene cage: Fig. 1(a)], cubic [[8]-benzene cage: Fig. 1(b)], and icosahedral [[20]-benzene cage: Fig. 1(c)] symmetries, respectively. Therefore, these cages are regarded as the tetrahedrane, cubane, and $\mathrm{C}_{20}-I_{h}$ fullerene with the inter- 
nal degree of freedom of benzene constituent unit. As for the two-dimensional network [Fig. 1(d)], each benzene is connected to its three adjacent, forming a hexagonal network of polymerized benzene. After optimization, these nanocages and the two-dimensional sheet retain their initial covalent network structures. The optimized bond lengths of the [4]-benzene cage are $1.42,1.61$, and $1.08 \AA$ for $d_{1}, d_{2}$, and $d_{\mathrm{H}}$, respectively [Fig. 1(e)]. For the [8]- and [20]-benzene cages, the optimized bond lengths are 1.41, 1.54, and 1.08 $\AA$ for $d_{1}, d_{2}$, and $d_{\mathrm{H}}$, respectively. Under the optimized structures, the benzene rings approximately keep a flat conformation with the buckled inter-benzene bonds $d_{2}$. Thus, with increase of the cage diameter, the bond length of $d_{2}$ monotonically decrease, reflecting the decrease of the strain. For the polymerized benzene, the optimized bond lengths are $1.41,1.50$, and $1.09 \AA$ for $d_{1}, d_{2}$, and $d_{\mathrm{H}}$, respectively. Thus, for any conformations, inter-benzene bond exhibit single bond nature as in the case of polyphenyl.

Figure 2 shows the total energy of the polymerized benzene nanocages and sheet as a function of their diameter. The energy is evaluated an equation,

$$
E=E_{t o t}-N_{\mathrm{C}} \mu_{\mathrm{C}}-N_{\mathrm{H}} \mu_{\mathrm{H}}
$$

where $E_{t o t}, N_{\mathrm{C}}, \mu_{\mathrm{C}}, N_{\mathrm{H}}$, and $\mu_{\mathrm{H}}$ are the total energy of nanocages or sheet, the number of $\mathrm{C}$ atoms, the chemical potential of $\mathrm{C}$ atom calculated using the total energy of graphene, the number of $\mathrm{H}$ atom, and the chemical potential of $\mathrm{H}$ atom, respectively. The total energy is approximately proportional in inverse square of the cage diameter, indicating that the $\pi$ electron are extended throughout the covalent networks. The calculated total energies of the [20]-benzene cage and the polymerized sheet are 0.88 and $0.78 \mathrm{eV} /$ atom, respectively, which is higher by twice than that of $\mathrm{C}_{60}$. Therefore, the materials have moderate energetic stability, even though they contain $\mathrm{C}-\mathrm{H}$ bonds which is less stable than $\mathrm{C}-\mathrm{C}$ bond. Therefore, once the materials have been synthesized, they keeps their cage or sheet structures under the ambient condition. Indeed, first principle MD simulations confirm their thermal stability up to the temperature of $1500 \mathrm{~K}$ for $1 \mathrm{ps}$ simulation times.

Figure 3 shows the electronic structure of the polymerized benzene nanocages and sheet. As for the nanocages, the electronic energy levels are bunched up owing to their high symmetry as well as the benzene constituent unit. In particular, many electron states are bunched up around the gap between the highest occupied (HO) and the lowest unoccupied (LU) states for the [20]-benzene cage. In contrast to the common electronic feature, detailed electronic structure depends on the cage size. The energy gap between 


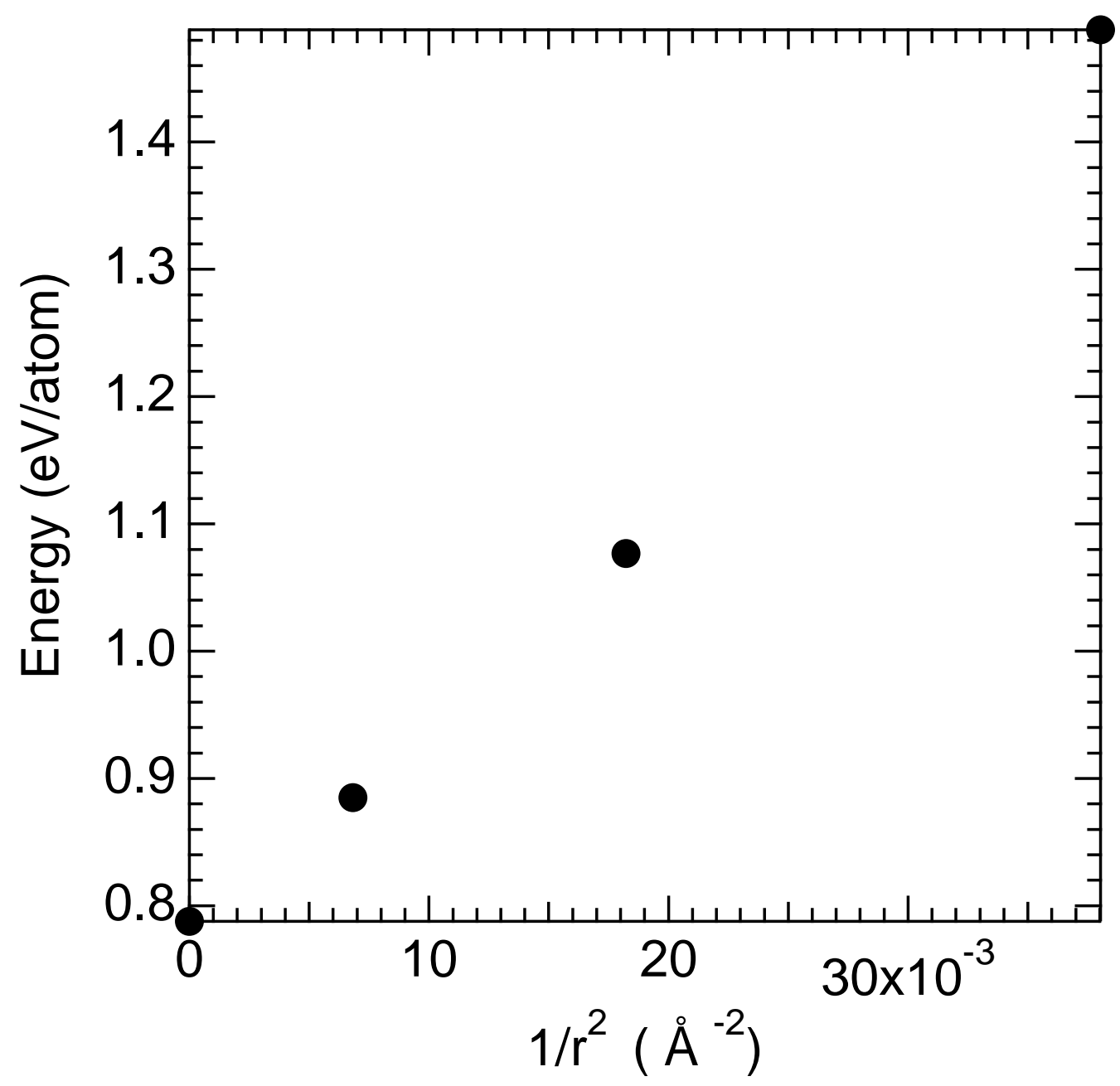

Fig. 2. The total energy of the polymerized [4]-, [8]-, [20]-benzene cages and that of the polymerized benzene sheet as a function of the inverse of square of their diameter.

$\mathrm{HO}$ and LU states is sensitive to the cage size: The calculated gap is 1.37, 1.59, and 2.33 $\mathrm{eV}$ for the [4]-, [8]-, and [20]-benzene cages, respectively, exhibiting monotonic increase of the gap with increasing the cage size. The increase of the gap is ascribed to decrease of the structural distortion arising from the curvature. Note that the gap of the [20]benzene cage is still narrower than that of an isolated benzene, because of the extended nature of $\pi$ electron throughout the cage.

Although the structural deformation arising from curvature is absent, the polymer has the band gap of $2.44 \mathrm{eV}$, which is still narrower than that of the gap between $\mathrm{HO}$ and LU states of benzene, because of the extended nature of $\pi$ electrons. In the case of two-dimensional benzene polymer, the network is a semiconductor with the direct gap 
(a)
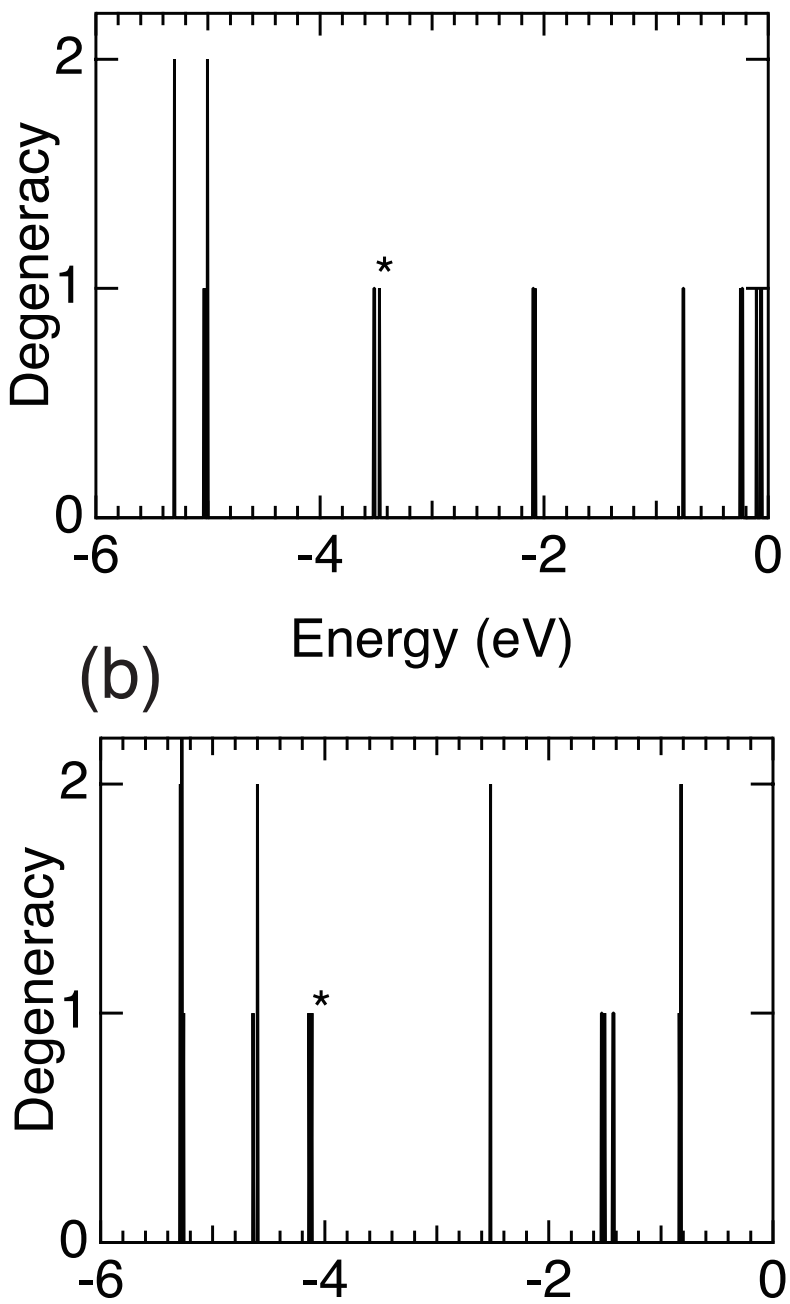

(c) Energy (eV)

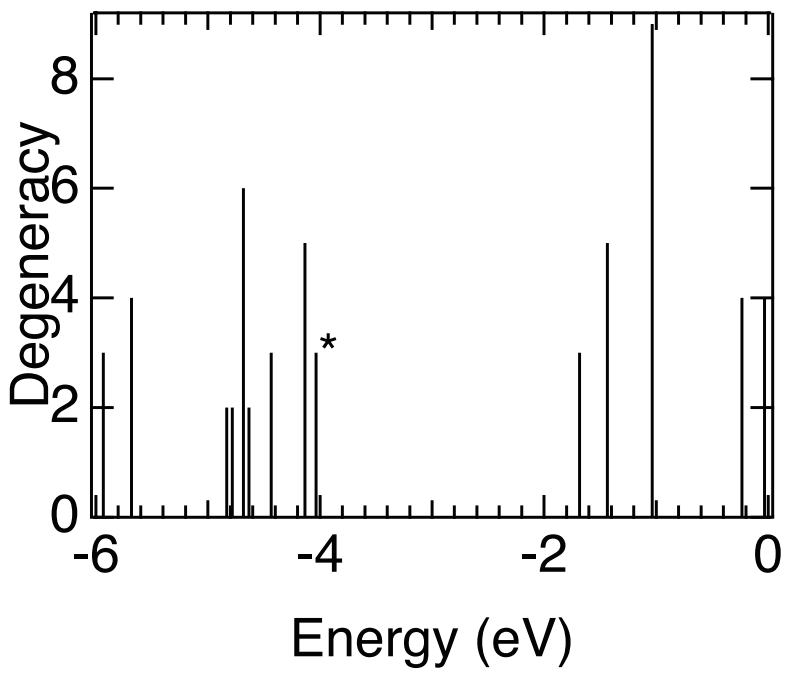

(d)

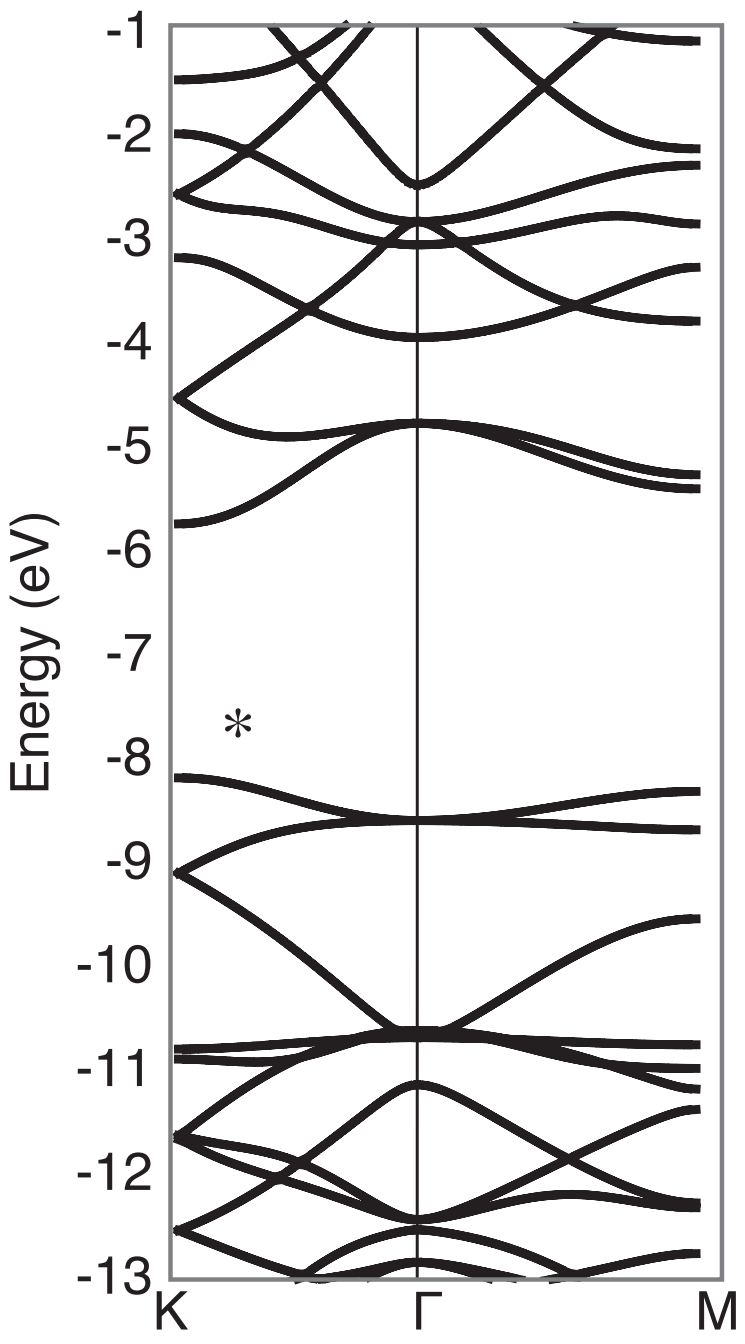

Fig. 3. Electronic structures of (a) [4]-, (b) [8]-, and (c) [20]-benzene cages and (d) that of the polymerized benzene sheet. An asterisk in each figure denotes the highest occupied states for the polymerized cages and the highest branch of the valence band for the polymerized sheet. The energy is evaluated from that of the vacuum level. 
at the K point. The polymer possesses approximately symmetric band structure with respect to the band gap as the case of the graphene and other hydrocarbon network, because of their symmetric topology in terms of their sub-lattice structure. On the other hand, the benzene polymer exhibits unusual band structure around its band edge: The highest branch of the valence band and the lowest branch of the conduction band exhibit their less dispersive nature compared with the other states. More interestingly, these less dispersive states degenerate with the other dispersive bands at $\Gamma$ point, which possess a linear dispersion relation around the $\mathrm{K}$ point, indicating that their kagome band feature.

Finally, we analyze the distribution of wave function of the HO and LU states of benzene cages and that of the highest branch of the valence band and the lowest branch of the conduction bands of the two-dimensional benzene polymer at the $\Gamma$ point. For the [4]- and [8]-benzene cages, because of the large structural deformation from the planar $\pi$ electron system, the wave function distribution is remarkably modulated from the $\mathrm{HO}$ and LU states of an isolated benzene molecule [Figs. 4(a) and 4(b)]. Thus, the modified distribution of wave function may cause the large HO-LU gap of the cages and the low-degeneracy around LU and HO states. In contrast, for the [20]-benzene cage and two-dimensional benzene polymer, HO and LU states possess the same distribution of the $\mathrm{HO}$ and LU states of the isolated benzene [Figs. 4(c) and 4(d)]. Furthermore, because of the covalent bonds connecting adjacent benzenes causes the interesting modulation in the HO and LU states. The HO state exhibits antibonding nature, while the LU state exhibits bonding nature with respect to the adjacent benzenes, indicating the fact that $\pi$ electron on benzene cage and polymer is extended throughout their covalent network as the case of graphene.

\section{Conclusion}

In this paper, we theoretically design the hierarchical nanoscale materials consisting of benzene as the potential nanomaterials those exhibit unusual electronic structure by conducting first-principles total-energy calculations based on the DFT with GGA. We proposed the possible nanostructures of [4]-, [8]-, and [20]-benzene cages which consist of 4,8 , and 20 benzene rings, respectively, each of which is connected its three adjacent via three of six edges of benzene. Furthermore, we consider the two-dimensional network of polymerized benzene as the graphene consisting of benzene. Accordingly, these materials are regarded as the tetrahedrane, cubane, $\mathrm{C}_{20}-I_{h}$ fullerene, and graphene 
(a)

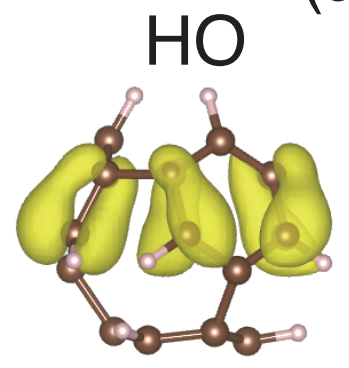

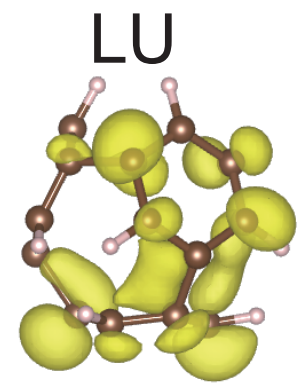

(c)

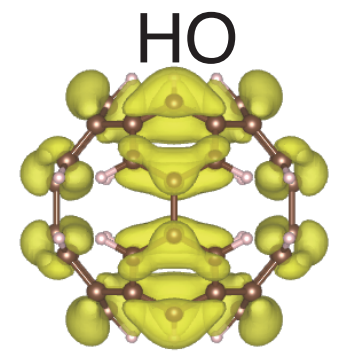

(b)

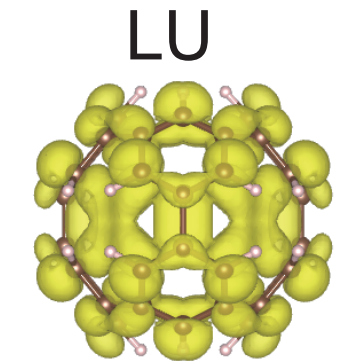

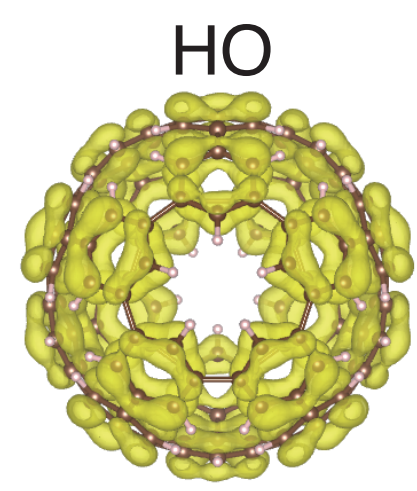

(d)

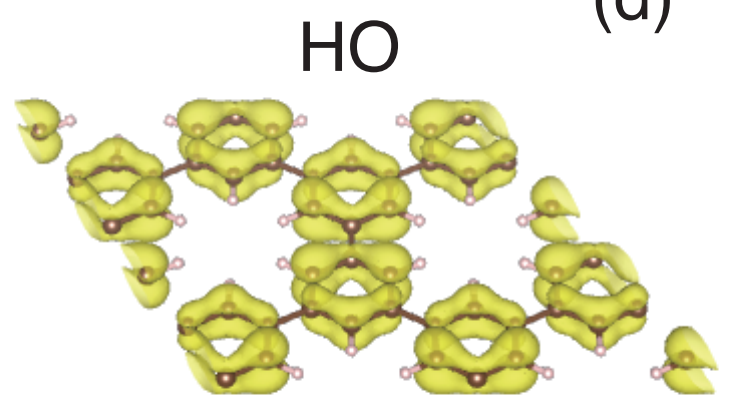

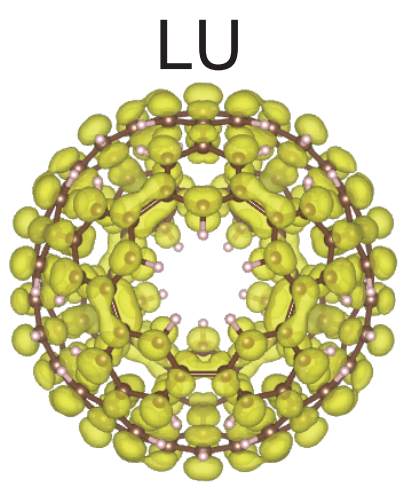

LU

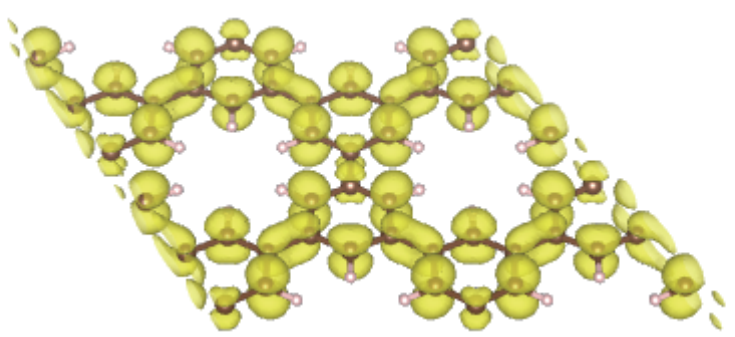

Fig. 4. (Color online) Squared wave function of the HO and LU states of (a) [4]-, (b) [8]-, (c) [20]-benzene cages and those of (d) the polymerized benzene sheet. For the polymer, the both wave functions are depicted at the $\Gamma$ point.

with the internal degree of freedom arising from benzene constituent unit. Our static and dynamical calculation on these materials corroborate the energetic a thermal stability of these materials. The nanocages have bunched electron states near their HO and LU states, because of the degenerated stats of constituent benzene. The two-dimensional benzene polymer is a semiconductor with the direct band gap at the $\mathrm{K}$ point with the gap energy of $2.44 \mathrm{eV}$, in spite of their flat conformation. By analyzing the wave function of these cages and polymer, we demonstrated that the $\pi$ electron near the HO-LU gap or the band edges is extended throughout the cages or sheet. 


\section{Acknowledgements}

This work was supported by JST-CREST Grant Numbers JPMJCR1532 and JPMJCR1715 from the Japan Science and Technology Agency, JSPS KAKENHI Grant Numbers JP17H01069, JP16H00898, and JP16H06331 from the Japan Society for the Promotion of Science, and the Joint Research Program on Zero-Emission Energy Research, Institute of Advanced Energy, Kyoto University. Part of the calculations was performed on an NEC SX-Ace at the Cybermedia Center at Osaka University and on an SGI ICE XA/UV at the Institute of Solid State Physics, The University of Tokyo. 


\section{References}

1) F. Bassani and G.P. Parravicini, Nuovo Cimento B 50, 95 (1967).

2) M. Posternak, A. Baldereschi, A. J. Freeman, E. Wimmer and M. Weinert, Phys. Rev. Lett. 50, 761 (1983).

3) E. Clar, The Aromatic Sextet (John Wiley and Sons, London, U.K. 1972).

4) Y. Morita, S. Suzuki, K. Sato, and T. Takui, Nature Chem. 3, 197 (2011).

5) V. Boekelheide and C.E. Larrabee, J. Am. Chem. Soc. 72, 1245 (1950).

6) D.H. Reid, Tetrahedron 3339 (1958).

7) K. Nakasuji, M. Yamaguchi, I. Murata, K. Yamaguchi, T. Fueno, H. Ohya-Nishiguchi, T. Sugano, and M. Kinoshita, J. Am. Chem. Soc. 1119265 (1989).

8) Z. Sun and J. Wu, J. Mater. Chem. 22, 4151 (2012).

9) N. Shima and H. Aoki, Phys. Rev. Lett. 71, 4389 (1993).

10) K. Kusakabe, K. Wakabayashi, M. Igami, K. Nakada, and M. Fujita, Mol. Cryst. Liq. Cryst. 305, 445 (1997).

11) M. Maruyama and S. Okada, Appl. Phys. Express 6, 095101 (2013)

12) M. Maruyama, N. T. Cuong, and S. Okada, Carbon 109, 755 (2016).

13) M. Maruyama and S. Okada, Carbon 125, 530 (2017)

14) N. Hamada, S. Sawada and A. Oshiyama, Phys. Rev. Lett. 68, 1579 (1992).

15) R. Saito, M. Fujita, M. S. Dresselhaus and G. Dresselhaus, Appl. Phys. Lett. 60, 2204 (1992).

16) K. Tanaka, K. Okahara, M. Okada and T. Yamabe, Chem. Phys. Lett. 191, 469 (1992).

17) M. Fujita, K. Wakabayashi, K. Nakada, and K. Kusakabe, J. Phys. Soc. Jpn. 65, 1920 (1996).

18) K. Nakada, M. Fujita, G. Dresselhaus, and M.S. Dresselhaus, Phys. Rev. B 54, 17954 (1996).

19) R. Jasti, J. Bhattacharjee, J. B. Neaton, and C. R. Bertozzi, J. Am. Chem. Soc. 130, 17646 (2008).

20) H. Takaba, H. Omachi, Y. Yamamoto, J. Bouffard, and K. Itami, Angew. Chem. Int. Ed. 48, 6112 (2009).

21) S. Yamago, Y. Watanabe, and T. Iwamoto, Angew. Chem. Int. Ed. bf 49, 757 (2010). 
$22)$ E. Kayahara, Vi. K. Patel, and S. Yamago, J. Am. Chem. Soc. 136, 2284 (2014).

23) P. J. Evans, E. R. Darz, and R. Jasti, Nature Chem. 6, 404 (2014).

24) H. A. Staab and F. Binnig, Tetrahedron Lett. 5, 319 (1964).

25) D. J. Cram, T. Kaneda, R.C. Helgeson, and G. M. Lein, J. Am. Chem. Soc. 101, $6752(1979)$.

26) D. J. Cram, T. Kaneda, R. C. Helgeson, S. B. Brown, and C. B. Knobler, E. Maverick, and K. N. Trueblood, J. Am. Chem. Soc. 107, 36451985.

27) W. Pisula, M. Kastler, C. Yang, V. Enkelmann, and K. Müllen, Chem.-Asian J. 2, 51 (2007).

28) J. M. W. Chan, and T. M. Swager,Tetrahedron Lett. 49, 4912 (2008).

29) J. Y. Xue, K. Ikemoto, N. Takahashi, T. Izumi, H. Taka, H. Kita, S. Sato, and H. Isobe, J. Org. Chem. 79, 9735 (2014).

30) M. O’Keeffe, G. B. Adams, and O. F. Sankey, Phys. Rev. Lett. 65, 2325 (1992).

31) K. Matsui, Y. Segawa, and K. Itami, J. Am. Chem. Soc. 136, 16452 (2014).

32) P. Li, L. N. Zakharov, and R. Jasti, Angew. Chem. Int. Ed. 56, 5237 (2017).

33) E. Kayahara, T. Iwamoto, H. Takaya, T. Suzuki, M. Fujitsuka, T. Majima, N. Yasuda, N. Matsuyama, S. Seki, and S. Yamago, Ntr. Commun. 4, 2694 (2013).

34) A. D. Finke and J. S. Moore, Org. Lett., 10, 4852 (2008).

35) K. Ikemoto, J. Lin, R. Kobayashi, S. Sato, and H. Isobe, Angew. Chem. Int. Ed. 57, 1 (2018).

36) M. Bieri, M. Treier, J. Cai, K. Aït-Mansour, P. Ruffieux, O. Gröning, P. Gröning, M. Kastler, R. Rieger, X. Feng, K. Müllen, and R. Fasel, Chem. Commun. 6919 (2009).

37) S. Kigure, H. Omachi, H. Shinohara, and S. Okada, J. Phys. Chem. C 119, 8931 (2015).

38) Y. Yamamoto, E. Tsurumaki, K. Wakamatsu, and S. Toyota, Angew. Chem. Int. Ed. 57, 8199 (2018).

39) P. Hohenberg and W. Kohn, Phys. Rev. 136, B864 (1964).

40) W. Kohn and L. J. Sham, Phys. Rev. 140, A1144 (1965).

41) Y. Morikawa, K. Iwata, and K. Terakura, Appl. Surf. Sci. 169-170, 11 (2001).

42) J. P. Perdew, K. Burke, and M. Ernzerhof, Phys. Rev. Lett. 77, 3865 (1997).

43) D. Vanderbilt, Phys. Rev. B 41, 7892 (1990). 